\title{
A Tentative Study of Hydrological and Ecological Parametric Remote Sensing Retrieval and Estimation
}

\author{
Yan $L i^{1, a}$ \\ ${ }^{1}$ Zhengzhou Vocational College of Industrial Safety, Zhengzhou University , Zhengzhou450000, \\ China \\ ayanzi@163.com
}

\begin{abstract}
Keywords: hydrological variable; ecological parameter; ecological remote sensing; estimation Abstract. In this article, a deep analysis and exploration is conducted on hydrological and ecological parametric remote sensing. Based on a brief analysis of hydrological variables and ecological parameters, the interaction between backscatter coefficient, polarization information and precipitation intensity is analyzed. In addition, to strengthen the accuracy of hydrological and ecological remote sensing studies in relevant regions, when discussing hydrological and ecological parametric retrieval and estimation, certain methods are combined to enhance the reliability of this article.
\end{abstract}

\section{Introduction}

At present, due to the limitation and constraint of a variety of conditions, there are rare analyses and studies on hydrological and ecological remote sensing in cold and arid regions in China. Studies on the interaction between hydrological and ecological parameters are often subject to topographic conditions, landscape characteristics and integrity. The effect of these factors in cold and arid regions is even more noticeable. Therefore, the analysis of hydrological and ecological parametric remote sensing retrieval and estimation in this article is of great guiding significance and practical value for the development and study of hydrological and ecological remote sensing in arid and cold regions.

\section{An Analysis of Hydrological Variables}

Hydrological variables mainly include precipitation, evapotranspiration, snow parameter, soil moisture and freeze-thaw on the earth's surface. For cold and arid regions, the spatial and temporal heterogeneity of precipitation are typical characteristics. So when analyzing the characteristics of river water circulation in such regions, it is very essential to obtain a high-resolution spatial and temporal distribution of precipitation. In this article, with Heihe River as an example, issues related to the hydrological and ecological parametric remote sensing retrieval and estimation in cold regions are illustrated. Research on the precipitation characteristics of the Heihe River is focused on its upstream and midstream. Vehicle-mounted X-band dual-polarization Doppler radar is used to make high-precision observation on precipitation in experimental areas, so as to analyze the relationship between precipitation intensity, backscatter coefficient and polarization information [1].

Through an analysis and observation of precipitation intensity, the following conclusions are drawn: 1. When regional differential characteristics of raindrop spectrum are analyzed, it is found that after differential transmission phase-shift constant is introduced, the precision of parameter formula should be higher than that of parameter formula under the effect of radar polarization and precipitation diameter; 2.The regional difference of raindrop spectrum is least influenced by raindrop spectrum; 3. There is a 6-power proportional relationship between backscatter coefficient and precipitation particle diameter. The differential reflectivity represents a 3-power proportional relationship with it. So backscatter coefficient is more sensitive to raindrop spectrum.

The significance of studying evapotranspiration lies in the final dissipation form of water. Therefore, the modeling and observation of evaporation is a key content of the studies on hydrological parameters in the Heihe River. An improved evapotranspiration parameterization scheme is to introduce a triangular space of earth's surface temperature and vegetation index during 
evapotranspiration estimation in study areas. Under automatic surface temperature conditions, rapid development and innovative applications have been achieved. The characteristics of different schemes are shown in Table 1 [2].

Table 1 The Evolution Process and Characteristics of Evapotranspiration Estimation Model

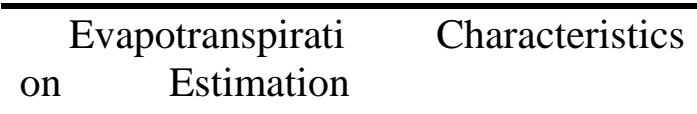

Scheme

\begin{tabular}{cl}
\hline $\begin{array}{c}\text { Iterative } \\
\text { algorithm }\end{array}$ & $\begin{array}{l}\text { Suitable for arid regions, can quantify and determine the dry } \\
\text { and wet sides of triangle space automatically. } \\
\text { In content, with MODIS as the data source and regional } \\
\text { sensible heat flux observations acquired by LAS as the basis, the } \\
\text { feasibility of the algorithm model is verified. }\end{array}$
\end{tabular}
parameterization scheme

Drag parameterization scheme

The developer is Xin. The use type: large arid regions with great temperature difference between canopy and earth’s surface. Difficulty in application: to obtain component temperature

By improving the reliability of parameterized canopy drag, improve the simulation precision of P-M model. Relevant factors include photosynthetically active radiation, root-zone soil moisture and temperature, etc.

Factors that affect the characteristic attributes of snow parameters include density, particle size, liquid water content, evaporation from snow surface and surface reflectivity and albedo, etc. In the process of observation, apart from these characteristic attributes, there are also characteristics to observe material balance component, intended to explore temporal and spatial variations in the process of energy balance. When different characteristics of snow parameters are observed, different observation methods will be selected. The details are shown in Table 2 [3].

Table 2 The Measuring Methods and Characteristics of Snow Parameters
Types of
Measuring Methods
Characteristics or Results
Detected

Characteristic

Parameters

Low spatial resolution,

Snow depth and To retrieve the snow depth limited when detecting snow snow water using a spaceborne passive depth and snow water equivalent microwave radiometer equivalent in mountainous regions

Snow grain size Layered snow microwave The overall simulation and density, etc. radiation model precision is good. During research, the effect of non-snow information on a sub-pixel scale must be considered.

Analysis of snow Snow spectrum and grain The spectrum dilution area grain size size data method plays a strong guiding role in the analysis of snow grain size

Studies on soil moisture generally adopt SAR data retrieval. The main purpose of this method is to reduce the effect of soil roughness on observations. With relevant technical researchers' optimization of SAR data retrieval, multi-angle ASAR observation data to retrieve soil moisture and surface roughness synchronously are formed. The analytical principle of this method is to establish an empirical formula between backscatter coefficient and soil roughness. 
Based on the above analysis of hydrological parameters, we can find that different hydrological parameters have diversified characteristic attributes. So in the process of analysis and test, different research methods must be selected. For key parametric attributes, the improved retrieval method and the remote sensing means must be combined.

\section{An Analysis of Ecological Parameters}

Ecological parameters mainly include vegetation classification, leaf area index, biomass and chlorophyll content, etc. In this article, several ecological parameters above are analyzed. Table 3 shows a comparison between the characteristics of C3 and C4 vegetation when ecological parameters of vegetation classification are studied. From data in this table, it can be seen that when a remote sensing classification method is adopted, the main basis is differences between C3 and C4 plants in chlorophyll fluorescence. On this basis, fluorescence under natural lighting of vegetation is obtained using Fraunhofer lines. They are classified in combination with vegetation functions. The classification precision of this method is up to $92.3 \%$ [4].

Table 3 The Differences between C3 and C4 Plants

\begin{tabular}{ccc}
\hline $\begin{array}{r}\text { Vegetation } \\
\text { Characteristic }\end{array}$ & $\begin{array}{c}\text { Fluorescen } \\
\text { ce Signal }\end{array}$ & $\begin{array}{c}\text { Chlorophyll } \\
\text { Fluorescence }\end{array}$ \\
\hline C3 & W & $7.8 \mathrm{~W}-2 . \mu \mathrm{m}-1$ \\
C4 & $2.2 \mathrm{~W}$ & $18.2 \mathrm{~W}-2 . \mu \mathrm{m}-1$ \\
\hline
\end{tabular}

Leaf area index mainly concerns the geometry of vegetation canopy. An important purpose of remote sensing application is to obtain leaf area index accurately. Table 4 shows basic links and content characteristics involved in the measurement of leaf area index using remote sensing means.

Table 4 Basic Links and Content Characteristics Involved in the Observation of Leaf Area Index

Basic Links to Realize Remote Sensing

Measurement of leaf The improved harvest measuring method improves the area index accuracy of measurement, with aggregation in fascicles, aggregation between fascicles and non-photosynthesis as the main influence factors.

Canopy-based To obtain multi-angle spectrum canopy reflectivity, radiation transmission with vegetation leaf reflectivity, transmittance and model multi-angle observation data as the input data to SALL

Retrieval of leaf area model. index

Scale effect

The non-linearity of retrieval method and spatial heterogeneity of leaf area index

The accuracy of biomass estimation directly affects the efficiency of light energy utilization (ELEU). As an important parameter that affects the accuracy of productivity model input, it is difficult to obtain the estimate of ELEU using traditional remote sensing means. Therefore, when estimating ELEU, it is necessary to use fluorescence information in the hyperspectral data to retrieve ELEU. Secondly, in the process of biomass estimation, methods, including LiDAR observation, eddy correlation system observation, as well as the parameter analysis method of ELEU model, etc., are adopted. In the process of analysis and observation, meteorological parameters must be combined to carry out a synthetic analysis and ensure that the biomass estimation has certain comprehensiveness and reliability [5].

Chlorophyll content observation is an important part in the study of chlorophyll characteristics. In the process of observation, continuous fine spectrum acquired by hyperspectral remote sensing is largely adopted. This observation method is also applicable to the accurate estimation of physiological and biochemical parameters, so it has wide applications and plays an important role in the research process of ecological parameters. After hyperspectral images are acquired, they can lay a 
foundation for the extraction of chlorophyll content and chlorophyll fluorescence intensity and also make it possible to conduct an ecological parametric analysis in arid regions and cold regions.

To sum up, the retrieval analysis of biological parameters in this article is an innovation and improvement on the basis of traditional retrieval means. The improved remote sensing means and retrieval tests depend on different techniques and biological principles. The predominant one is to introduce hyperspectral characteristic images. Through a spectral analysis, information about different characteristic parameters of different biological parameters can be effectively grasped, to observe and extract relevant information.

\section{Conclusion}

Given the above, this article mainly includes two aspects. A remote sensing and retrieval analysis is conducted on elements related to hydrological and ecological parameters. Observation and remote sensing means under different observation attributes are analyzed. Through a comprehensive analysis, it is found that under the effect of mechanical lidar-radar and high-resolution optical images, high-precision surface feature information can be acquired and the feasibility to apply laser radar technology in vegetation structure detection is exploited. When exploring leaf area index, it is found that the new method of retrieving ELEU can be applied to fluorescence information in hyperspectral data.

\section{References}

[1]Quattrochi D A, Luvall J C. Thermal infrared remote sensing for analysis of landscape ecological processes: methods and applications[J]. Landscape Ecology, 1999, 14(6):577-598.

[2]Jian Y. Prediction of Mosquito Abundance in Temperate Regions, Using Ecological, Hydrological and Remote Sensing Models[J]. Dissertations \& Theses - Gradworks, 2014.

[3]Tang Q H, Gao H L, Lu H, et al. Remote sensing: hydrology.[J]. Progress in Physical Geography, 2009, 33(4):490-509.

[4]Santi E, Paloscia S, Pettinato S, et al. Comparison between SAR Soil Moisture Estimates and Hydrological Model Simulations over the Scrivia Test Site[J]. Remote Sensing, 2013, 5(10):4961-4976.

[5]Zhixiao Xie, Leonard Pearlstine, Dale E. Gawlik. Developing a Fine-Resolution Digital Elevation Model to Support Hydrological Modeling and Ecological Studies in the Northern Everglades[J]. Giscience \& Remote Sensing, 2012, 49(5):664-686. 